\title{
is Research Square \\ Improved Muscle Function After Injury with the Application of a Biological Decellularized Matrix
}

Amina El Ayadi ( $\sim$ amelayad@utmb.edu )

University of Texas Medical Branch at Galveston https://orcid.org/0000-0002-3657-0633

Melody R.S. Threlkeld

University of Texas Southwestern Medical Center at Dallas

Steven E. Wolf

University of Texas Medical Branch

Juquan Song ( $\sim$ jusong@utmb.edu )

University of Texas Medical Branch

\section{Research}

Keywords: muscle isometric force, porcine-derived urinary bladder matrix (UBM), laceration injury

Posted Date: June 1st, 2020

DOI: https://doi.org/10.21203/rs.3.rs-31848/v1

License: (c) (7) This work is licensed under a Creative Commons Attribution 4.0 International License. Read Full License 


\section{Abstract}

Background: Skeletal muscle injury leads to loss of muscle function that lasts well into recovery and can be permanent. Application of the novel bio-scaffold termed porcine-derived urinary bladder matrix (UBM) has a potential benefit to mitigate injury through tissue regeneration. To date, findings of potential benefit in animal models were limited to short assessment times. The purpose of this study was to investigate whether UBM treatment 14 days after injury sustainably improves the recovery of muscle function in injured mice.

Methods: C57BL/ 6 adult male mice received bilateral laceration injuries on the gastrocnemius (GN) muscle under anesthesia and were then treated with vehicle or $150 \mu \mathrm{g}$ of UBM nanoparticles. Treatment was applied immediately after injury or 14 days later. Muscle isometric force was measured 60 days after injury. Previous time course analyses have shown that muscle function did not start to improve until after 42 days after injury. Therefore, we designed a second experiment to trace the time course of UBM effects on muscle function recovery by measuring the isometric muscle force at 49 and 90 days after injury. In vitro, we analyzed the effects of UBM on muscle cell proliferation and differentiation.

Results: UBM promotes muscle cell proliferation and differentiation. Twitch (Pt), tetanic (Po) force and maximal fatigue were significantly decreased in the injured mice on day 60. Muscle fatigue maximum force significantly recovered when UBM treatment was applied 14 days after injury $(p<0.05)$ but not when UBM was applied immediately after the injury. Time course analysis demonstrated that UBM improvement of Pt and Po was evident by day 49 after injury $(p<0.05)$. However, no further muscle function improvement was observed on day 90.

Conclusions: Delayed treatment with the UBM improves muscle function recovery following laceration injury starting 49 days after injury. These effects may be mediated by improvements in muscle cell proliferation and differentiation. This animal model is suitable to test other therapeutic strategies to improve muscle function after injury.

\section{Background}

Skeletal muscle represents the largest tissue mass in the body, accounting for $40-45 \%$ of total body weight [1]. Loss of skeletal muscle from direct injury can present significant debilitating effects that threaten the full recovery of function. For those with loss of muscle from direct injuries, such as projectile fragments or crush injury, current management options include functional free muscle transfer and the use of advanced bracing [2], which have limited efficiency on functional recovery. Recent reports describing functional free muscle transplantation in the forearm [3] and elbow [4] have limitations with insufficient muscle functional recovery. Improvement in muscle regeneration is becoming a more suitable option for patients with severe muscle injuries or muscle loss.

Novel regenerative medicine approaches have been tested in clinical studies for skeletal muscle regeneration in trauma and burn patients [5-7]. Aside from studies of cell viability, the extracellular environment is considered one of the most important factors related to the regeneration of functional tissue. The extracellular matrix provides a robust structural microenvironment for cell growth and contains factors that can stimulate cell migration and differentiation into functional tissue components [8]. The extracellular matrix derived from decellularized porcine urinary and bladder mucosa (UBM) [9] was shown to improve the regeneration of various types of tissue in vivo [10,11]. UBM contains over $90 \%$ type I, III, IV and V collagens and other components including glycosaminoglycan, transforming growth factor, basic fibroblast growth factor, and vascular endothelial growth factor. UBM provides a promising microenvironment for regeneration and contains putative bio-active growth factors for progenitor cell recruitment and propagation [12]. 
A clinical case report on severely injured patients with volumetric muscle loss showed significant improvements in muscle mass and function with implantation of porcine-derived extracellular matrix [13]. In the above-mentioned report, the isokinetic performance was significantly enhanced four months after implantation. The formation of new tissue was also evident with computer tomography [13]. Muscle mass increased by $15 \%$ and muscle function improved by over $20 \%$ when comparing leg extension torque data obtained 5 months before UBM implantation to data obtained 9 months after [13]. Another clinical study revealed that UBM improvement of muscle function was associated with enrichment in innervated muscle tissue islands [14]. However, the mechanisms by which UBM treatment improves muscle regeneration are still to be investigated.

We sought to use a suitable preclinical model to address these clinical questions. Most preclinical studies using animal models of muscle injury use matrix treatments immediately after injury $[15,16]$, which does not fully mimic clinical settings and thus limit data interpretation and translation. In general, healing of injured tissue and recovery is affected by the acute inflammatory response after injury, subacute repair phase, and late remodeling phase. Muscle function outcome depends on the stage when treatment is applied. This study was designed to determine the effects of UBM on muscle function when applied during or after the acute phase of injury. Injured animals were treated with UBM nanoparticles immediately or 14 days after injury (Fig. 1). Muscle isometric function was examined at days 49, 60 and 90 after injury.

\section{Methods}

\section{In vitro studies}

We first analyzed the ability of UBM to induce muscle cell proliferation. C2C12 muscle cells (ATCC, Manassas, VA) were grown in regular growth media containing Dulbecco's Modified Eagle Media (DMEM), 10\% FBS, and 1\% glutamine. The cells were then treated with $0,5,50$, or $250 \mu \mathrm{g} / \mathrm{ml}$ of powder UBM (Acell Inc, Columbia, MD) for 18 hours. The cell lysates were then collected and the protein expression of proliferating cell nuclear antigen (PCNA) was determined using western blotting as previously described [17]. Briefly, cell lysates were stored on ice for 20 minutes and then centrifuged at 13,000 $\mathrm{g}$ for 20 minutes. Protein concentration was determined by the bicinchoninic acid method (Thermo-Scientific, Waltham, MA). Next, $30 \mu \mathrm{g}$ lysate was mixed with $2 \times$ sodium dodecyl sulfate (SDS) sample buffer and separated on 4-20\% Tris-glycine gels (Biorad Laboratories, Hercules, CA) by SDS-polyacrylamide gel electrophoresis. Proteins were transferred onto polyvinylidene fluoride membranes, blocked with $5 \%$ bovine serum albumin (BSA) in 1\% Tris-buffered saline plus 0.5\% Tween 20 (TBS-T), and incubated with primary antibodies in 5\% BSA in TBS-T overnight at $4{ }^{\circ} \mathrm{C}$. Blots were washed in TBS-T and incubated with anti-rabbit or mouse horseradish peroxidase secondary antibody at room temperature for 1 hour. Blots were visualized using enhanced chemiluminescence substrates for the detection of horseradish peroxidase enzyme activity (Thermo Scientific, Waltham, MA). The primary antibody for PCNA was obtained from Cell Signaling Technology (Danvers, MA). Betaactin was used as a loading control. Band quantification was performed using ImageJ software (available at NIH.org http://imagej.nih.gov/ij/), and values were normalized to the loading control.

\section{Immunostaining}

We choose the $250 \mu \mathrm{g} / \mathrm{ml}$ dose of UBM that showed the highest effects on muscle cell proliferation to determine the effects of UBM on the expression of myogenic markers Pax-7 in $\mathrm{C} 2 \mathrm{C} 12$ cells. Following 18 hours of treatment, the cells were fixed with methanol and immunostaining for Pax-7 was conducted following established protocols [17].

\section{Animal studies}


C57BL/ 6 male adult mice (7 weeks old, from Jackson Laboratory, Bar Harbor, ME) were acclimated for one week in the animal facility at the University of Texas Southwestern Medical Center (UTSW). All experimental procedures followed $\mathrm{NIH}$ guidelines and were approved by the Institutional Animal Care and Use Committee (IACUC) at UTSW. Mice were housed individually with free access to water and food during the experiment.

Experiment 1 was designed to compare the effects of early versus delayed treatment with UBM on muscle function recovery after injury. Twenty mice received bilateral laceration injuries to the gastrocnemius muscle under anesthesia and were randomized to an immediate treatment group $(E)$, or a delayed treatment group by 14 days after injury $(L)$ (Fig. 1A). At each time point, injured animals were further grouped by treatment with either vehicle $(V, n=4)$ or $U B M(U$, $n=6)$. Four additional animals did not receive any intervention and served as controls.

Experiment 2 was designed to test the temporal effect of UBM treatment on muscle function over time. Sixteen mice received bilateral laceration injuries to the gastrocnemius muscle under general anesthesia and were randomly treated with vehicle $(\mathrm{V}, \mathrm{n}=8)$ or UBM $(\mathrm{U}, \mathrm{n}=8) 14$ days later. Previous studies from Walters' group showed that improvement in muscle function was not seen until 42 days after injury [28]. Therefore, we designed this experiment to trace earlier time points when UBM may affect muscle function. Four animals from each group were examined for function recovery at day 49 or day 90 after injury (Fig. 1B). Eight additional animals did not receive any procedure and served as controls ( $\mathrm{n}=4$ per time point).

\section{Muscle laceration procedure}

Mice were weighed then anesthetized with $2 \%$ isoflurane inhalation. The hair was removed from both hind limbs while on a prone position in aseptic conditions. The skin above the gastrocnemius muscle was incised using a surgical blade (no. 11; SteriSharps, Mansfield, MA). The gastrocnemius was bluntly isolated from adjacent biceps femoris and semitendinosus. GN was then separated from soleus by inserting a steel scalpel handle (Bard-Parker, size 4) and two parallel full muscle thickness incisions ( $3 \mathrm{~mm}$-width and $7 \mathrm{~mm}$-length) were made longitudinally in the middle of GN (Fig. 1). Whole pieces of muscle were excised with fine scissors to induce an estimated $20 \% \mathrm{GN}$ tissue loss. After laceration, the skin was closed with a simple interrupted suture. Buprenorphine-SR $0.05 \mathrm{mg} / \mathrm{kg}$ was administered to reduce any pain or discomfort. Fully recovered animals were housed individually with food and water ad libitum.

\section{UBM/Vehicle treatment}

MatriStem MicroMatrix (UBM) was purchased from ACell Inc. (Columbia, MD). Mice were anesthetized with $2 \%$ isoflurane. UBM $(150 \mu \mathrm{g})$ was suspended in $15 \mu \mathrm{l}$ of DMEM (ATCC, Manassas, VA) and injected intramuscularly using a $1 \mathrm{ml}$ syringe with $23 \mathrm{~g} \mathrm{3/4}$ needle (BD Bioscience, San Jose, CA). DMEM was used as a vehicle and $15 \mu \mathrm{L}$ DEM was administered to the vehicle-treated animals. Once ambulatory, the animals were returned to their home cage and housed individually until the end of the experiment.

\section{Isometric force measurement}

Isometric contractile properties of the GN were measured using the Aurora 1305A whole animal system (Aurora Scientific, ON, Canada) as described previously [18]. Under general anesthesia, mice were secured on the $37^{\circ} \mathrm{C}$ platform connected to a circulating water bath. GN muscles of the left lower limb were gently dissected free of surrounding tissue and the Achilles tendon was secured with 4 - 0 surgical suture and attached to the lever arm of a dual-mode servo muscle lever system (mod \#305c, Aurora Scientific, Inc). Electrodes were implanted into the distal end of the severed sciatic nerve and the twitch ( $\mathrm{Pt})$, tetanic $(\mathrm{Po})$ and fatigue isometric functions of the muscle were analyzed after optimal length (Lo) of the muscle was achieved. A single Pt was stimulated with a $200 \mathrm{~Hz}$ impulse at $10 \mathrm{~mA}$ and the muscle was then stretched $0.2 \mathrm{~mm}$ every 25 seconds. The Lo of the muscle was reached when less than $2 \%$ change was obtained between twitches. The isometric Po force was stimulated at $150 \mathrm{~Hz}$ with impulse 
duration of 0.2 milliseconds, 75 pulses per train for a total of 1 second. Fatigue measurement was at $40 \mathrm{~Hz}$ with an impulse duration of $0.2 \mathrm{~ms}, 14$ pulses per train, $10 \mathrm{~mA}$, for a total of 240 seconds.

\section{Muscle sample harvest and histology}

Animals were euthanized after in situ muscle function testing, and GN from both limbs was collected and weighed. Muscle tissues were fixed in 10\% neutral buffered formalin and histologically processed for paraffin embedding.

Tissue sections $(5 \mu \mathrm{m})$ were deparaffinized and rehydrated then stained following the Masson's Trichrome staining protocol. Images were taken by a Nikon Eclipse Ti microscope under a 10X objective using NIS-Elements v4.60 software.

\section{Statistical analysis}

Statistical analysis was performed using two-way analysis of variance (ANOVA) with Bonferroni post hoc tests and paired Student's t-test where appropriate. All data are presented as mean \pm standard error of the mean (SEM); significance was accepted at $p<0.05$.

\section{Results}

\section{Effect of UBM on muscle cell proliferation and differentiation}

UBM was applied to C2C12 cells at increasing concentrations of $0,2,50$, and $250 \mu \mathrm{g} / \mathrm{ml}$. We found that UBM increases the protein expression of the PCNA with the maximum effect seen with the $250 \mu \mathrm{g} / \mathrm{ml}$ dose $(p<0.05)$ (Fig. 2A). We then chose the $250 \mu \mathrm{g} / \mathrm{ml}$ concentration to determine the effect of UBM on C2C12 differentiation and found that UBM increases the protein expression of Pax7 in murine myoblasts (Fig. 2B).

\section{Effect of UBM on muscle force at 60 days}

After normalizing to the mouse body weight at day 0 , we found no significant effect of treatment on the mice body weight at day 60 (Table 1). We also corrected the tissue weight of GN muscle (at day 60) by adding measured excised tissue weight, then normalizing to body mass. We found that the corrected tissue weight was greater in animals that received the treatment on day 14 compared to those treated immediately after injury $(p<0.05)$, suggesting a potential benefit for delayed UBM treatment (Table 1). 
Table 1

Mice whole body weight and muscle weight changes 60 days after injury.

\begin{tabular}{|c|c|c|c|c|c|c|}
\hline $\begin{array}{l}\text { Treatment time after } \\
\text { injury }\end{array}$ & Treatment & $\begin{array}{l}\text { Group } \\
\text { (n) }\end{array}$ & $\begin{array}{l}\text { Body weight } \\
\text { change }\end{array}$ & TW (g) & $\begin{array}{l}\text { TW/BM } \\
(\mathrm{mg} / \mathrm{g})\end{array}$ & $\begin{array}{l}\text { Corrected } \\
\text { TW/BM (mg/g) }\end{array}$ \\
\hline No injury & No & $\begin{array}{l}\mathrm{N}-\mathrm{C}(\mathrm{n} \\
=5)\end{array}$ & $1.18 \pm 0.01$ & $0.138 \pm 0.002$ & $5.21 \pm 0.11$ & $5.21 \pm 0.11$ \\
\hline \multirow[t]{2}{*}{0 day $(E)$} & Vehicle & $\begin{array}{l}E-V(n \\
=4)\end{array}$ & $1.16 \pm 0.02$ & $0.091 \pm 0.009$ & $3.41 \pm 0.33$ & $4.39 \pm 0.45$ \\
\hline & UBM & $\begin{array}{l}\text { E-U }(n \\
=6)\end{array}$ & $1.17 \pm 0.02$ & $0.092 \pm 0.003$ & $3.47 \pm 0.09$ & $4.24 \pm 0.09$ \\
\hline \multirow[t]{2}{*}{14 days $(\mathrm{L})$} & Vehicle & $\begin{array}{l}\mathrm{L}-\mathrm{V}(\mathrm{n} \\
=4)\end{array}$ & $1.21 \pm 0.01$ & $0.110 \pm 0.011$ & $4.14 \pm 0.49$ & $5.13 \pm 0.43$ \\
\hline & UBM & $\begin{array}{l}\operatorname{L-U}(\mathrm{n} \\
=6)\end{array}$ & $1.19 \pm 0.05$ & $0.102 \pm 0.007$ & $3.84 \pm 0.23$ & $5.04 \pm 0.13 \Delta \mu$ \\
\hline
\end{tabular}

$\Delta, \mathrm{p}<0.05$, late (L) vs. Earlier treatment $(\mathrm{E}) ; \mu, \mathrm{p}<0.05$, L-U vs. E-U group, two-way ANOVA. TW-Gastrocnemius tissue weight; BM-body weight at day 60 after injury.

The Pt, Po, and fatigue isometric functions were analyzed in situ on day 60 after injury with muscle stretched to optimal length. Pt was significantly decreased in injured mice at day $60(78.14 \pm 7.69 \mathrm{~g})$ compared to normal mice $(101.50 \pm 3.74 \mathrm{~g})(\mathrm{p}<0.05)$. Po was also reduced in injured mice at day $60(184.62 \pm 9.34 \mathrm{~g})$ compared to normal mice $(272.96 \pm 17.84 \mathrm{~g})(\mathrm{p}<0.05)$. The optimal length (Lo) was also significantly higher when animals were treated with UBM on day 14 after injury, compared to immediate treatment $(p<0.05)$. Muscle maximal fatigue force (Ft-max) was higher in mice treated 14 days after injury $(176.12 \pm 14.85)$ compared to immediately treated $(127.47 \pm 18.38)$ and vehicle-treated animals $(108.96 \pm 19.00)(p<0.05)($ Table 2$)$. 
Table 2

Isometric force in the mouse right gastrocnemius 60 days after injury.

\begin{tabular}{|c|c|c|c|c|c|}
\hline \multicolumn{2}{|l|}{ D60 } & \multicolumn{2}{|l|}{ INJURY + } & \multicolumn{2}{|l|}{ INJURY + } \\
\hline & \multirow{3}{*}{$\begin{array}{l}\text { No injury, No } \\
\text { treatment N-C } \\
(\mathrm{n}=5)\end{array}$} & \multicolumn{2}{|c|}{ Treatment at 0 day } & \multicolumn{2}{|c|}{ Treatment at 14 days } \\
\hline & & Vehicle & UBM & Vehicle & UBM \\
\hline & & $E-V(n=4)$ & $E-U(n=6)$ & $\mathrm{L}-\mathrm{V}(\mathrm{n}=4)$ & $L-U(n=6)$ \\
\hline $\begin{array}{l}\text { Tissue } \\
\text { weight (g) }\end{array}$ & $0.152 \pm 0.010$ & $0.143 \pm 0.009$ & $0.122 \pm 0.005$ & $0.124 \pm 0.007 *$ & $0.132 \pm 0.008$ \\
\hline Lo (cm) & $1.51 \pm 0.04$ & $1.54 \pm 0.097$ & $1.55 \pm 0.04$ & $1.75 \pm 0.04^{\star}$ & $1.54 \pm 0.05 \varphi$ \\
\hline 1/2 RT (s) & $0.0165 \pm 0.0016$ & $0.0142 \pm 0.0006$ & $0.01656 \pm 0.0008$ & $0.0156 \pm 0.0009$ & $0.0156 \pm 0.0009$ \\
\hline Pt (g) & $101.50 \pm 3.74$ & $78.14 \pm 7.69+$ & $83.91 \pm 3.88$ & $72.24 \pm 5.96+$ & $89.57 \pm 4.60$ \\
\hline Po (g) & $272.96 \pm 17.84$ & $235.93 \pm 7.44$ & $212.36 \pm 23.43$ & $184.62 \pm 9.34+$ & $230.95 \pm 16.87$ \\
\hline Pt/Po & $0.36 \pm 0.01$ & $0.32 \pm 0.02$ & $0.40 \pm 0.02$ & $0.37 \pm 0.03$ & $0.44 \pm 0.03$ \\
\hline sPo/PCSA & $282.68 \pm 18.33$ & $270.74 \pm 30.89$ & $282.08 \pm 33.38$ & $293.99 \pm 37.85$ & $279.03 \pm 13.99$ \\
\hline Ft-max & $178.56 \pm 27.08$ & $133.19 \pm 16.90$ & $127.47 \pm 18.38$ & $108.96 \pm 19.00$ & $176.12 \pm 14.85 \varphi \Delta$ \\
\hline \multicolumn{6}{|c|}{$\begin{array}{l}+, p<0.05 \text {, vs. N-C, Student's t-test. * }{ }^{*}, p<0.05 \text {, vs. E-V; } \Delta, p<0.05 \text {, vs. E-U; } \varphi, p<0.05 \text {, vs. L-V, two-way ANOVA. TW- } \\
\text { tissue weight; Lo- optimal length; } 1 / 2 \text { RT-half relaxation time; Pt- twitch force; Po- tetanic force; sPo-specific tetanic } \\
\text { force; Ft-Fatigue }\end{array}$} \\
\hline
\end{tabular}

\section{Time course effect of UBM on muscle force}

Since the beneficial effects of delayed treatment were observed in the first experiments, we sought to determine the time course of delayed UBM treatment on muscle function. Animals were treated with UBM 14 days after injury and muscle function examined at 49 and 90 days after injury. Isometric force readings for Pt and Po were significantly decreased in injured mice at day 49 compared to non-injured control mice $(p<0.05)$. Delayed treatment with UBM significantly preserved muscle function parameters $\mathrm{Pt}, \mathrm{Po}$, and specific tetanic force (sPo) when compared to vehicletreated mice $(p<0.05)$ (Table 3$)$. These results confirmed the beneficial effects of delayed treatment on day 14 observed in Experiment 1. At day 90, we found no difference in body weight and tissue weight between injured and non-injured mice. Besides this, no additional change of muscle function was observed between testing at 60 and 90 days (Table 4). 
Table 3

Isometric force in the mouse right gastrocnemius 49 days after injury in Experiment 2.

\begin{tabular}{|c|c|c|c|}
\hline \multirow[t]{3}{*}{ D 49} & & \multicolumn{2}{|c|}{ Injury and Treatment } \\
\hline & No treatment & Vehicle & UBM \\
\hline & $\mathrm{N}-\mathrm{C}(\mathrm{n}=4)$ & $V(n=4)$ & $\mathrm{U}(\mathrm{N}=4)$ \\
\hline $\mathrm{BM}(\mathrm{g})$ & $27.14 \pm 0.88$ & $25.01 \pm 0.96$ & $26.33 \pm 0.20$ \\
\hline TW (g) & $0.171 \pm 0.007$ & $0.143 \pm 0.001+$ & $0.150 \pm 0.006$ \\
\hline TW/BM (mg/g) & $6.28 \pm 0.20$ & $5.75 \pm 0.21$ & $5.68 \pm 0.20$ \\
\hline Lo (cm) & $1.69 \pm 0.07$ & $1.55 \pm 0.08$ & $1.52 \pm 0.05$ \\
\hline 1/2 RT (s) & $0.0156 \pm 0.002$ & $0.0142 \pm 0.001$ & $0.0143 \pm 0.001$ \\
\hline $\mathrm{Pt}(\mathrm{g})$ & $91.39 \pm 2.84$ & $61.21 \pm 4.18+$ & $82.85 \pm 7.24 \Delta$ \\
\hline Po (g) & $324.52 \pm 49.54$ & $251.25 \pm 16.53+$ & $300.16 \pm 4.29 \Delta$ \\
\hline $\mathrm{sPo}\left(\mathrm{N} / \mathrm{cm}_{2}\right)$ & $34.06 \pm 5.21$ & $24.36 \pm 2.05$ & $30.86 \pm 1.83 \Delta$ \\
\hline Ft-max (g) & $176.25 \pm 17.02$ & $147.48 \pm 13.33$ & $163.97 \pm 17.58$ \\
\hline Ft-min (g) & $8.88 \pm 0.64$ & $9.25 \pm 4.09$ & $8.37 \pm 3.18$ \\
\hline
\end{tabular}

Table 4

Isometric force in mouse right gastrocnemius 90 days after injury in Experiment 2.

\begin{tabular}{|c|c|c|c|}
\hline \multirow[t]{3}{*}{ D 90} & No injury & Treatment & UBM \\
\hline & No treatment & Vehicle & $U(n=4)$ \\
\hline & $\mathrm{N}-\mathrm{C}(\mathrm{n}=4)$ & $V(n=4)$ & \\
\hline Body mass & $31.41 \pm 1.17$ & $30.11 \pm 1.17$ & $28.84 \pm 1.46$ \\
\hline TW left (g) & $0.192 \pm 0.006$ & $0.157 \pm 0.015$ & $0.164 \pm 0.0016$ \\
\hline TW/BM $(\mathrm{mg} / \mathrm{g})$ & $6.13 \pm 0.29$ & $5.21 \pm 0.46$ & $5.67 \pm 0.43$ \\
\hline Lo (mm) & $16.5 \pm 0.3$ & $15.8 \pm 0.7$ & $15.0 \pm 0.9$ \\
\hline $1 / 2$ RT (s) & $0.0141 \pm 0.0006$ & $0.0148 \pm 0.0005$ & $0.0132 \pm 0.0004$ \\
\hline $\mathrm{Pt}(\mathrm{g})$ & $68.59 \pm 6.14$ & $54.24 \pm 5.20$ & $44.59 \pm 5.92$ \\
\hline Po (g) & $175.64 \pm 31.85$ & $163.76 \pm 42.89$ & $154.69 \pm 45.78$ \\
\hline sPo (N/cm2) & $20.68 \pm 2.57$ & $14.64 \pm 3.34$ & $16.20 \pm 4.82$ \\
\hline Ft-max & $72.92 \pm 13.17$ & $83.43 \pm 29.08$ & $63.27 \pm 28.35$ \\
\hline Ft-min & $3.42 \pm 0.65$ & $6.16 \pm 3.56$ & $3.06 \pm 1.34$ \\
\hline
\end{tabular}




\section{UBM reduces muscle fibrosis}

When analyzed 60 days after injury, collagen accumulation was still evident in the GN muscle of injured mice that were treated immediately after injury compared to vehicle-treated animals. Collagen accumulation was seen in both the vehicle and the early UBM treated animals. However, collagen accumulation was reduced in the GN muscle of injured mice that were treated with UBM 14 days after injury (Fig. 3).

\section{Discussion}

The preclinical model presented in this study with direct muscle laceration and excision shows that muscle mass loss leads to impairment in muscle function with over $23 \%$ decrease in maximum Po force when examined 60 days after injury. This decrease was not significant at a later time point (90 days after injury). We further found that treatment with UBM nanoparticles improved muscle function starting day 49 after injury.

Many preclinical models of direct injury are used to study muscle regeneration, including cryoinjury [19], chemical cardiotoxin injection [20], physical contusion [21], and sharp laceration [22]. Wu et al. [22] developed the rat model of massive muscle loss with laceration and excision and fully described the healing time process. We chose this model with a similar laceration and excision injury to test the effects of UBM on muscle regeneration.

Using a similar model, Merritt et al. [23] observed that injured rats with homologous extracellular matrix implantation presented only some tissue morphologic improvements but maintained about $20 \%$ force loss at 42 days after injury. However, no improvement was observed when examined at earlier time points. We, thus, designated our initial observational time at 60 days after injury. We did not observe any significant changes with the treatment applied immediately after injury (E-U vs. E-V). Surprisingly, an increased fatigue maximum force was observed in injured mice at 60 days when UBM was applied 14 days after injury. To trace the time course for the effect of UBM on muscle function, we also analyzed isometric muscle force at day 49 after application and found significant improvements in twitch force, tetanic force, and specific tetanic force.

The beneficial effect of UBM on muscle function was diminished at day 90 after injury, which may be due to biomaterial depletion and subsequent regenerative limitation. UBM was formulated as absorbable nanoparticles and contains several growth factors. When UBM is metabolized and depleted of growth factors, the tissue regenerative effects from the applied dose may be limited. We did not test the effects of multiple applications, which could be considered in future studies.

We also noted that the force values at day 90 were lower compared to other time points and that might be due to the system calibration before each experiment. Animal posture and its relationship to the transducer could also affect the reading values. Our pilot data showed slight differences in isometric muscle force between the left and right limbs from the same animal. Therefore, we chose in this study to measure muscle force only in the left limb. Non-injury and no-treatment animals were tested as controls to validate each experiment.

In the current study, we focused on the physiological improvements of the GN muscle by assessing the isometric force. Beattie et al. [24] reported that UBM treatment improved tendon repair, which was associated with chemotaxis of multi-potential progenitor cells to the wound site; this benefit might be related to the bioactive components released from UBM [25]. Though we did not focus on the cellular mechanisms underlying the improvement of muscle function with UBM application, we speculate that the time of UBM intervention could be critical since no distinguishable cell population was identified during the muscle recovery period. The role of immune cells, specifically macrophages, is important in this healing process [26]. The delayed application of UBM may match the time of phenotypic transition of 
macrophages or monocytes after injury, further facilitating muscle regeneration and improvement of function. Our next step will be to investigate how UBM interacts with M1 and M2 macrophages during the muscle recovery period.

The cellular mechanism of UBM mediation of muscle regeneration and recovery is complex. Goldman et al. [27] reported that UBM worsens co-grafted muscle cells in the rat model at 2 and 8 weeks after injury. Another study from Walters' group showed that the biological matrix had no effect on muscle cells, but the effects were primarily on fibroblasts [28]. The same report showed that placement of extracellular matrix immediately after injury improved muscle function and fibrotic tissue remodeling, but there was no evidence of myofiber regeneration at day 28 [28]. We previously observed increased myogenesis in a cryo-injured mouse with UBM treatment [29]. In vitro, we found that UBM increases the protein expression of the proliferation marker PCNA in the mouse myoblast C2C12 cells, supporting a direct effect of UBM on muscle cells (Fig. 2). Trichrome staining showed prominent fibrosis in injured muscle tissue that was attenuated by a delayed UBM treatment but not with early treatment. Meanwhile, centrally localized myotubes were observed in the UBM-treated group (Fig. 3). The different outcomes between the various studies might be explained by differences in the experimental settings, especially the treatment time. Here, we applied the nanoparticle form of UBM 14 days after injury. The specific treatment condition could alter the interaction between different types of cells involved in muscle tissue regeneration.

\section{Conclusions}

In conclusion, the current study provides a reliable and clinically relevant model to investigate therapeutic approaches to improve muscle function recovery. The data from the current study showed that UBM nanoparticles accelerate muscle function recovery in injured mice. Furthermore, the limitation in the long-term effects of UBM on muscle function warrants more investigations to optimize this therapeutic strategy specifically the time and number of applications.

\section{Abbreviations}

Bovine serum albumin (BSA)

Dulbecco's Modified Eagle Media (DMEM)

Gastrocnemius (GN)

Optimal length (Lo)

Proliferating cell nuclear antigen (PCNA)

Sodium dodecyl sulfate (SDS)

Tetanic (Po)

Tween 20 (TBS-T)

Twitch (Pt)

Urinary bladder matrix (UBM)

\section{Declarations}




\section{Competing interests}

The authors declare that they have no competing interests.

\section{Funding}

This work was supported by funds from the Golden Charity Guild Charles R. Baxter, MD, Chair; the Department of Defense \#W81XWH-13-1-0462; and the National Institute of General Medical Sciences under award number T32GM008593.

\section{Authors' Contributions}

AE participated in experimental design, data analysis, and manuscript preparation. MRST performed animal experiments. SEW participated in experimental design from a clinical perspective and manuscript preparation. JS participated in experimental design, data collection, data analysis, and manuscript preparation.

\section{Ethics Approval}

All experimental procedures were followed by NIH guidelines and were approved by the Institutional Animal Care and Use Committee (IACUC) at the University of Texas Southwestern Medical Center (UTSW).

\section{Availability of Data and Materials}

The datasets used during the current study are available from the corresponding author upon request.

\section{Acknowledgments}

We thank Kevin DeSpain for supporting data collection. We also thank Eileen Figueroa and Steve Schuenke from UTMB Health for their help with manuscript preparation.

\section{References}

1. Beatty $\mathrm{CH}$, Basinger $\mathrm{GM}$, Bocek RM. Differentiation of red and white fibers in muscle from fetal, neonatal and infant rhesus monkeys. J Histochem Cytochem. 1967;15:93-103.

2. Lin CH, Lin YT, Yeh JT, et al. Free functioning muscle transfer for lower extremity posttraumatic composite structure and functional defect. Plast Reconstr Surg. 2007;119:2118-26.

3. Fan C, Jiang P, Fu L, et al. Functional reconstruction of traumatic loss of flexors in forearm with gastrocnemius myocutaneous flap transfer. Microsurgery. 2008;28:71-5.

4. Vekris MD, Beris $A E$, Lykissas $M G$, et al. Restoration of elbow function in severe brachial plexus paralysis via muscle transfers. Injury. 2008;39:S15-22.

5. Novikova LN, Novikov LN, Kellerth JO. Biopolymers and biodegradable smart implants for tissue regeneration after spinal cord injury. Curr Opin Neurol. 2003;16:711-5. 
6. Schertzer JD, Lynch GS. Comparative evaluation of IGF-I gene transfer and IGF-I protein administration for enhancing skeletal muscle regeneration after injury. Gene Ther. 2006;13:1657-64.

7. Burd A, Ahmed K, Lam S, et al. Stem cell strategies in burns care. Burns. 2007;33:282-91.

8. van der Kraan PM, Buma P, van Kuppevelt T, et al. Interaction of chondrocytes, extracellular matrix and growth factors: relevance for articular cartilage tissue engineering. Osteoarthritis Cartilage. 2002;10:631-7.

9. Brown BN, Barnes CA, Kasick RT, et al. Surface characterization of extracellular matrix scaffolds. Biomaterials. 2010;31:428-37.

10. Gilbert TW, Gilbert S, Madden M, et al. Morphologic assessment of extracellular matrix scaffolds for patch tracheoplasty in a canine model. Ann Thorac Surg. 2008;86:967-74.

11. Liu L, Li D, Wang Y, et al. Evaluation of the biocompatibility and mechanical properties of xenogeneic (porcine) extracellular matrix (ECM) scaffold for pelvic reconstruction. Int Urogynecol J. 2011;22:221-7.

12. Hodde JP, Record RD, Tullius RS, et al. Retention of endothelial cell adherence to porcine-derived extracellular matrix after disinfection and sterilization. Tissue Eng. 2002;8:225-34.

13. Mase VJ, Jr., Hsu JR, Wolf SE, et al. Clinical application of an acellular biologic scaffold for surgical repair of a large, traumatic quadriceps femoris muscle defect. Orthopedics. 2010;33:511.

14. Dziki J, Badylak S, Yabroudi M, et al. An acellular biologic scaffold treatment for volumetric muscle loss: results of a 13-patient cohort study. NPJ Regen Med. 2016;1:16008.

15. Sicari BM, Rubin JP, Dearth CL, et al. An acellular biologic scaffold promotes skeletal muscle formation in mice and humans with volumetric muscle loss. Sci Transl Med. 2014;6:234ra58.

16. Corona $\mathrm{BT}, \mathrm{Wu} \mathrm{X}$, Ward $\mathrm{CL}$, et al. The promotion of a functional fibrosis in skeletal muscle with volumetric muscle loss injury following the transplantation of muscle-ECM. Biomaterials. 2013;34:3324-35.

17. El Ayadi A, Stieren ES, Barral JM, et al. Ubiquilin-1 regulates amyloid precursor protein maturation and degradation by stimulating K63-linked polyubiquitination of lysine 688. Proc Natl Acad Sci U S A. 2012;109:13416-21.

18. Saeman MR, DeSpain K, Liu MM, et al. Severe burn increased skeletal muscle loss in mdx mutant mice. J Surg Res. 2016;202:372-9.

19. Warren GL, Hulderman T, Mishra D, et al. Chemokine receptor CCR2 involvement in skeletal muscle regeneration. FASEB J. 2005;19:413-5.

20. Gayraud-Morel B, Chretien F, Flamant $P$, et al. A role for the myogenic determination gene Myf5 in adult regenerative myogenesis. Dev Biol. 2007;312:13-28.

21. Crisco JJ, Jokl P, Heinen GT, et al. A muscle contusion injury model. Biomechanics, physiology, and histology. Am J Sports Med. 1994;22:702-10.

22. Wu X, Corona BT, Chen X, et al. A standardized rat model of volumetric muscle loss injury for the development of tissue engineering therapies. Biores Open Access. 2012;1:280-90.

23. Merritt EK, Hammers DW, Tierney M, et al. Functional assessment of skeletal muscle regeneration utilizing homologous extracellular matrix as scaffolding. Tissue Eng Part A. 2010;16:1395-405.

24. Beattie AJ, Gilbert TW, Guyot JP, et al. Chemoattraction of progenitor cells by remodeling extracellular matrix scaffolds. Tissue Eng Part A. 2009;15:1119-25.

25. Reing JE, Zhang L, Myers-Irvin J, et al. Degradation products of extracellular matrix affect cell migration and proliferation. Tissue Eng Part A. 2009;15:605-14.

26. Wynn TA, Vannella KM. Macrophages in tissue repair, regeneration, and fibrosis. Immunity. 2016;44:450-62. 
27. Ehrhart IC, Parker PE, Weidner WJ, et al. Coronary vascular and myocardial responses to carotid body stimulation in the dog. Am J Physiol. 1975;229:754-60.

28. Aurora A, Roe JL, Corona BT, et al. An acellular biologic scaffold does not regenerate appreciable de novo muscle tissue in rat models of volumetric muscle loss injury. Biomaterials. 2015;67:393-407.

29. Song J, Hornsby P, Stanley M, et al. Porcine urinary bladder extracellular matrix activates skeletal myogenesis in mouse muscle cryoinjury. 2014. http://www.hoajonline.com/jrmte/2050-1218/3/3. Accessed 30 March 2020.

\section{Figures}

\section{Study Design}

A Muscle Laceration Procedure

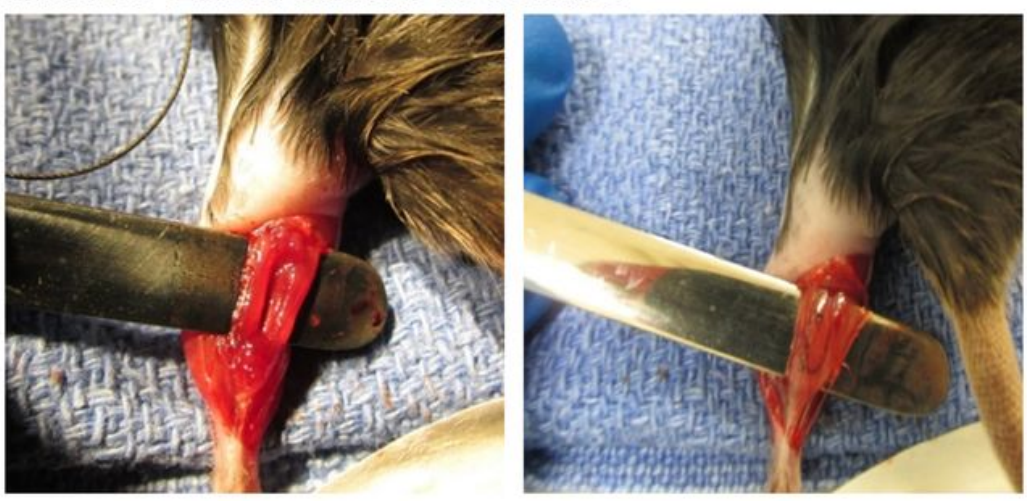

\section{B Experiment 1}

d1 Muscle Laceration

d1 UBM or Vehicle

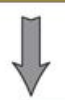

\section{Experiment 2}

d1 Muscle Laceration

d1 UBM or Vehicle

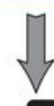

d49 Isometric Force Measurement

d14 UBM or Vehicle d49 Muscle Tissue Harvest

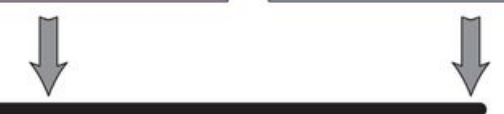

\section{Figure 1}

Animal experiment design and muscle laceration procedure. A) Muscle laceration procedure; B) Experiment 1; C) Experiment 2. 
A
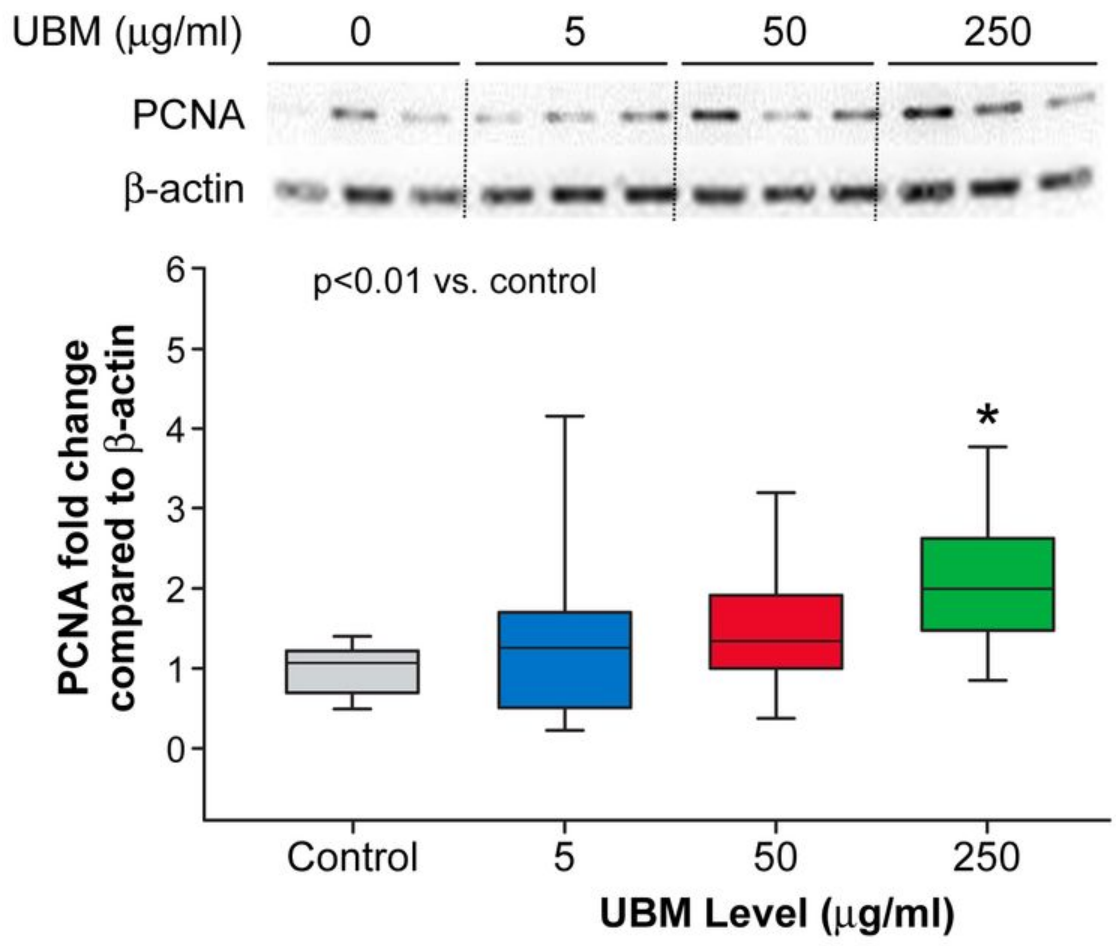

\section{Control}

UBM $(250 \mu \mathrm{g} / \mathrm{ml})$

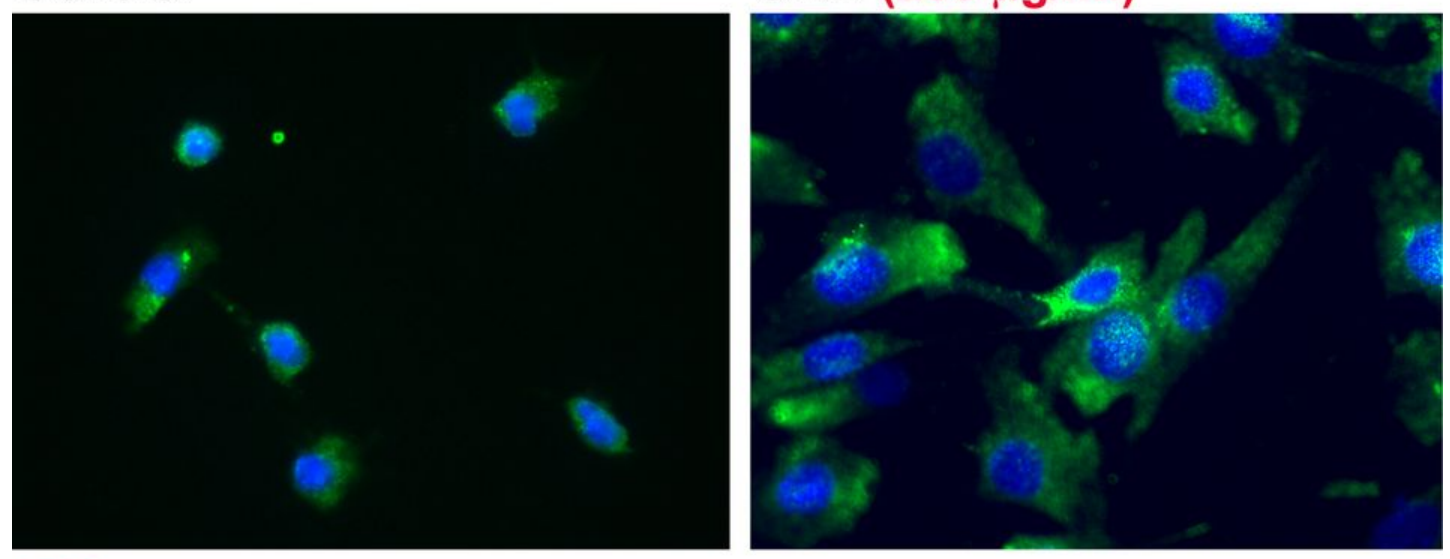

IFC staining with DAPI (blue) Pax7 (green)

Figure 2

Effect of UBM on muscle cell proliferation and differentiation. C2 C12 cells were treated with increasing concentrations of $\operatorname{UBM}(0,5,50.250 \mathrm{ug} / \mathrm{ml})$ in DMEM growth media to determine (A) PCNA protein expression by western blotting, and (B) Pax-7 immunostaining. DAPI (blue) Pax7 (green). Data are the mean \pm SEM. * $p<0.01$ vs. control 


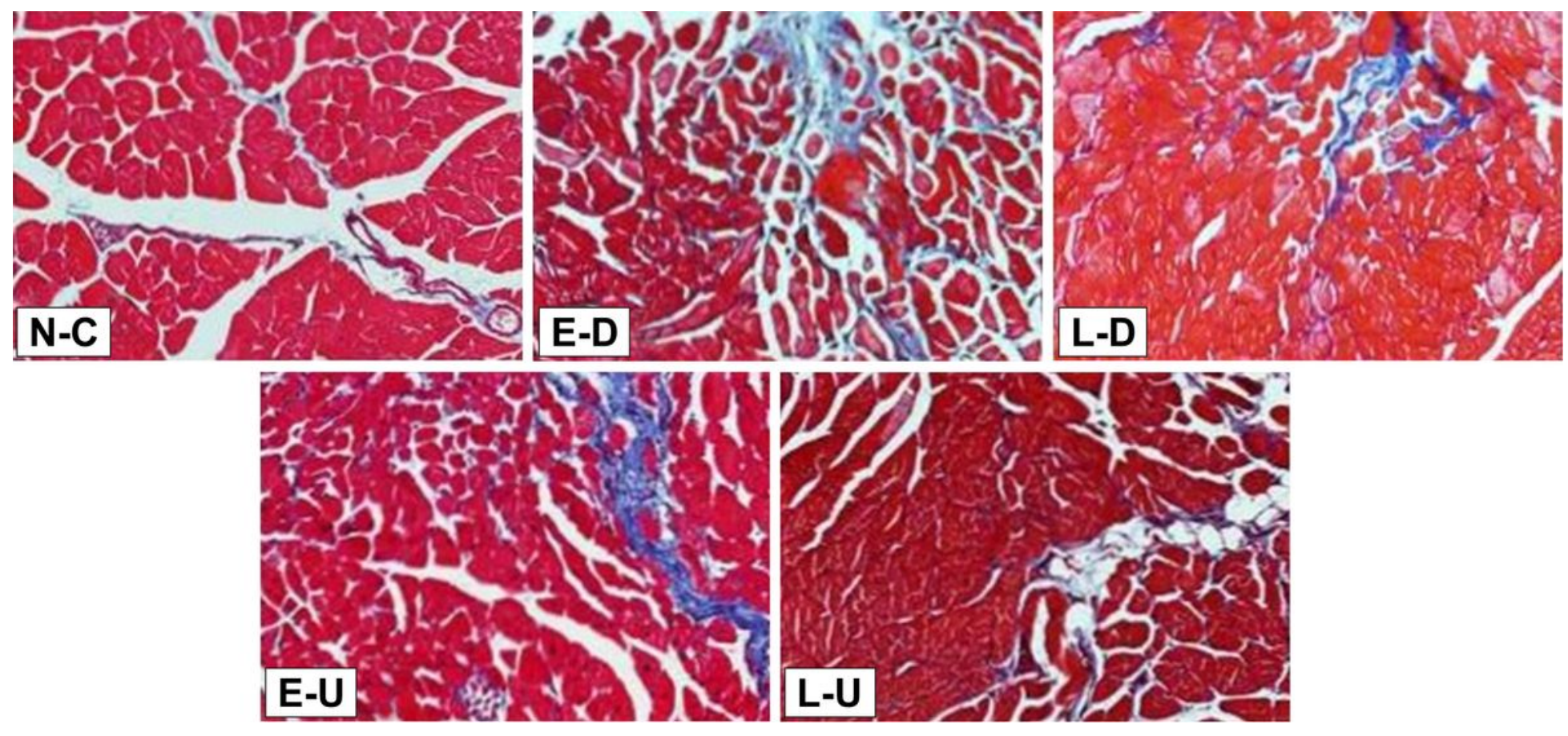

Figure 3

UBM reduces injury-induced fibrosis in the GN muscle. Trichrome staining of mouse GN muscle. N-C: normal control; ED: DMEM treatment immediately after injury; E-U: UBM treatment immediately after injury; L-D: DMEM treatment at 14 days after injury; L-U: DMEM treatment at 14 days after injury. 